\title{
ACTUALIDAD POLÍTICA Y CONSTITUCIONAL FRAN- CESA (2007-2008): ¿HACIA LA V REPÚBLICA BIS?
}

\author{
ÁNGEL J. SÁNCHEZ NAVARRO \\ Profesor Titular de Derecho Constitucional \\ Universidad Complutense de Madrid
}

\author{
SUMARIO \\ I. Introducción \\ II. Las reformas constitucionales de 2008 \\ III. A modo de conclusión
}

\section{INTRODUCCIÓN}

Probablemente no resulte exagerado afirmar que en los diez años transcurridos desde el nacimiento de esta revista, y con ella de estas crónicas sobre la vida política e institucional francesa, una de las principales características de la misma ha sido la proliferación de reformas constitucionales. De hecho, tales reformas centraban ya la primera de ellas, que constataba «una considerable aceleración del ritmo" de las mismas hasta el punto de justificar el fenómeno que Louis Favoreu denominó "banalización" de la reforma constitucional, a partir del dato de que la Constitución de 1958 había sufrido cinco reformas, de diverso calado, desde su entrada en vigor hasta 1991; y seis más entre 1992 y aquel año de $1998^{1}$.

Pues bien: desde entonces hasta nuestros días, como cualquier seguidor de estas páginas habrá podido comprobar, tal fenómeno no sólo no se ha atenuado sino que, por el contrario, se ha intensificado. En efecto, desde 1999 hasta la primera mitad de 2007 han tenido lugar otras once reformas del texto constitucional francés, afectando a cuestiones muy diversas que van desde las suscitadas por el progresivo proceso de integración europea (1999, 2003,

1 Vide TRC, núm. 1 (1998), págs. 205 y ss. 
2005), hasta la reciente consagración constitucional de la abolición de la pena de muerte (2007), pasando por otras como la paridad electoral (1999), la reducción del mandato presidencial (2000), la organización descentralizada de la República (2003) o la responsabilidad del Presidente de la misma (2007). Por ello el propio Consejo Constitucional, en su página web, señala que «la Constitución debe constituir un marco estable que no se modifique más que de forma solemne y por buenas [sérieuses] razones", por lo que "la repetición de las reformas, desde 1992, resulta preocupante [un peu préoccupante], ${ }^{2}$.

En todo caso, la tendencia no parece remitir en absoluto. Y así, durante el último año, y mientras la atención que los medios españoles prestan a Francia parece exclusivamente centrada en la mediática figura de su Presidente, dos nuevas reformas han venido a sumarse a la lista, elevando hasta 24 su número total (diecinueve desde 1992). La primera de ellas, en línea con otras anteriores, respondía a las nuevas circunstancias que atraviesa el proceso de integración europea, tras el fracaso del Tratado por el que se establece una Constitución para Europa. Sin embargo, y como trataremos de mostrar en las páginas que siguen, la segunda presenta características particulares que la hacen especialmente destacable. Es más: prima facie, parece ser la reforma de mayor calado de la Constitución gala desde que, en 1962, se estableció la elección del Presidente de la República mediante sufragio universal. De ahí que haya llegado a hablarse de la VRepública bis ${ }^{3}$.

\section{LAS REFORMAS CONSTITUCIONALES DE 2008}

\section{LA ADAPTACiÓN AL NUEVO MARCO EUROPEO}

Una vez más, la primera reforma ha venido impuesta por las vicisitudes del proceso de integración europea. Concretamente, en esta ocasión el elemento desencadenante ha sido el rechazo del llamado Tratado constitucional europeo por los propios ciudadanos franceses quienes, al pronunciarse con sus votos en contra del mismo, dejaron sin efecto la labor realizada por la Ley constitucional 2005-204, de 1 de marzo de 2005, que daba al Título XV de la Constitución una nueva redacción llamada a surtir efectos «a partir de la entrada en vigor del Tratado" ${ }^{4}$.

Como es bien sabido, la respuesta a ese fracaso - agravado por el inmediato voto negativo de los holandeses - ha sido la firma de un nuevo Tratado: el "Tratado de Lisboa por el que se modifican el Tratado de la Unión

2 Cfr.www.conseil-constitutionnel.fr/textes/modif.htm

3 Véanse, por ejemplo, Libération (15 de julio de 2008); o el editorial de Le Figaro del 22 del mismo mes.

4 Sobre el debate constitucional provocado por el Tratado, el contenido de la citada Ley constitucional y los principales datos del referéndum, vid. TRC, núms. 15 (2005) y 16 (2006), páginas 365-383 y 397-398, respectivamente. 
Europea y el Tratado Constitutivo de la Comunidad Europea, firmado en Lisboa el 13 de diciembre de 2007.5. Lo cual, lógicamente, ha obligado a adecuar nuevamente la redacción del citado Título XV de la Constitución gala.

De esta forma, el día 4 de febrero del presente año la reunión conjunta en Congreso de ambas Cámaras aprobó la correspondiente Ley constitucio$\mathrm{nal}^{6}$. Una Ley que obtuvo el respaldo de una mayoría holgada, y más que suficiente, de los parlamentarios, pero en absoluto un apoyo generalizado ${ }^{7}$. Es más: aunque en distinto grado, la mayor parte de los grupos votaron como, por otra parte, es habitual en el Parlamento francés- divididos ${ }^{8}$.

En cuanto a su contenido, y según resulta de cuanto se ha expuesto, la reforma adquiere una dimensión esencialmente técnica, sin perjuicio de sus obvias repercusiones políticas. Así, la Ley modifica el epígrafe del Título XV, que - siempre a partir de la entrada en vigor del Tratado de Lisboa- pasará a denominarse "De la Unión Europea", abandonando pues la fórmula anterior ("De las Comunidades Europeas y de la Unión Europea"), adoptada en 1992. Y, a continuación, ajusta la redacción de los artículos 88-1 y siguientes de la Constitución al nuevo marco de Lisboa: sólo el 88-3 permanece inalterado. En todo caso, algunas de estas disposiciones (concretamente, los artículos 88-4 y

5 DOCE, 2007/C 306/01, de 17 de diciembre. Un Tratado que, hasta la fecha, ha sido ratificado por la inmensa mayoría de los Estados miembros de la Unión, encontrando no obstante un nuevo obstáculo, derivado del resultado negativo de otro referéndum, esta vez en Irlanda (junio de 2008). tution.

6 Loi constitutionnelle n. ${ }^{\circ}$ 2008-103 du 4 février 2008 modifiant le titre XV de la Consti-

7 Recuérdese que, de acuerdo con el artículo 89 de la Constitución, la aprobación de la reforma por le Congrès du Parlement requiere una mayoría de tres quintos de los votos emitidos. En este caso el texto, tras ser aprobado, en los mismos términos, por la Asamblea Nacional (16 de enero) y por el Senado ( 29 de enero), fue sometido al Congreso el 4 de febrero. Allí, de los 893 parlamentarios que participaron en la votación, 152 optaron por abstenerse; en consecuencia, los votos emitidos fueron 741 y la mayoría requerida era de 445 votos. Finalmente, los votos favorables se impusieron por 560 frente a 181 .

8 Como en otras ocasiones recientes, la división afectó fundamentalmente a los Grupos Socialistas (en la Asamblea Nacional, "Grupo Socialista, Radical, Ciudadano y de independientes de izquierda"). Entre sus parlamentarios, la mayoría optó por la abstención ( 94 diputados y 49 senadores, entre los cuales se encuentran nombres bien conocidos como los de François Hollande, Pierre Mauroy, Pierre Bourguignon, Elisabeth Guigou o Daniel Vaillant), mientras que votaron a favor de la reforma 17 diputados y 15 senadores (entre ellos, Jack Lang o Robert Badinter), y se opusieron 91 diputados y 30 senadores (Laurent Fabius, Henri Emmanuelli...). Pero también se encuentra en los demás grupos: así, entre aquellos más favorables a la reforma, los parlamentarios de la mayoritaria UMP optaron abrumadoramente por el voto afirmativo (307 diputados y 150 senadores), si bien 7 votaron en contra (3 diputados y 4 senadores, entre ellos, Charles Pasqua), y 5 más se abstuvieron. Los centristas (Nuevo Centro, en la Asamblea; y Unión Centrista-UDF, en el Senado) también se inclinaron casi unánimemente por el "sín" (20 diputados y 30 senadores), aunque 2 diputados se opusieron. Y otro tanto cabe decir del Grupo senatorial RDSE (15 a favor, 1 en contra). Por el contrario, los grupos de la izquierda más radical (Gauche Démocrate et Républicaine en la Asamblea, y Communiste, Républicain et Citoyen en el Senado) votaron muy mayoritariamente en contra ( 21 diputados y 23 senadores), con sólo 3 abstenciones entre sus diputados. Finalmente, de los "no inscritos" votaron a favor 3 diputados y 3 senadores (entre los primeros, François Bayrou); en contra, 4 y 2, respectivamente; y otro senador optó por abstenerse. 
88-5) se han visto ya modificadas por la ulterior reforma constitucional de 21 de julio (modernización de las instituciones). En este marco, y salvo que señalemos otra cosa, nos limitaremos a recoger aquí los textos resultantes de la primera modificación, dejando para más tarde las aportaciones de la segunda.

De este modo, el artículo 1 de la Ley dispone que, en el segundo párrafo del artículo 88-1, la referencia al Tratado constitucional de 2004 se sustituye por otra al Tratado de Lisboa. Además, desde la entrada en vigor de este último los actuales dos párrafos de este artículo quedarán refundidos en uno, según el cual "La República participa en la Unión Europea, compuesta por Estados que han optado libremente por ejercer en común algunas de sus competencias en virtud del Tratado de la Unión Europea y del Tratado de Funcionamiento de la Unión Europea, conforme al texto modificado por el Tratado de Lisboa de 13 de diciembre de 2007" (artículo 2.2 $2^{\circ}$ de la Ley).

El mismo artículo $2.2^{\circ}$ prescribe a continuación que, igualmente a partir de la entrada en vigor del Tratado de Lisboa, el artículo 88-2 pierde sus dos primeros párrafos actuales (introducidos, en respuesta a los Tratados de Maastricht y Amsterdam, por las reformas constitucionales de 1992 y 1999) ${ }^{9}$. Y el tercero (también incorporado en 2003, a fin de permitir la puesta en marcha de la orden europea de detención y entrega ${ }^{10}$ ) pierde asimismo su actual referencia al Tratado de la Unión Europea, que pasa a hacerse a "las instituciones" de la misma. De esta manera, el artículo 88-2 quedará redactado como sigue: "Se establecerán por ley las normas sobre la orden de detención europea, en aplicación de los actos aprobados por las instituciones de la Unión Europea".

Por su parte, el artículo 88-4 (cuya redacción, derivada de las reformas de 1992 y 1999, se ha modificado tanto en febrero como en julio de 2008), queda redactado en los siguientes términos: "El Gobierno someterá a la Asamblea Nacional y al Senado, en el momento de su transmisión al Consejo de la Unión Europea, los proyectos o proposiciones de actos de las Comunidades Europeas y de la Unión Europea". El párrafo segundo prevé, también desde julio, que "Del modo que se establezca en el Reglamento de cada Cámara, podrán adoptarse resoluciones europeas, incluso fuera de los períodos de sesiones, sobre los proyectos o proposiciones mencionados en el párrafo anterior, así como sobre cualquier documento emanado de una institución de la Unión Europea ${ }^{11}$. En cualquier caso, la vigencia del Tratado de Lisboa de-

9 Sobre estas reformas, vid. TRC, núms. 1 (1998) y 3 (1999), págs. 209-210 y 236-240, respectivamente.

10 Vid. TRC, núms. 12-13 (2003-2004), págs. 484-486.

11 De acuerdo con la reforma de febrero, este artículo disponía que "El Gobierno someterá a la Asamblea Nacional y al Senado, en el momento de su transmisión al Consejo de la Unión Europea, los proyectos de actos legislativos europeos, así como los demás proyectos o propuestas de actos de la Unión Europea que contengan preceptos sobre materias de reserva de ley. Podrá igualmente someter a las Cámaras otros proyectos o propuestas de actos, así como cualquier documento, que emane de una institución de la Unión Europea". En su párrafo segundo, no modificado en febrero, se decía que "Del modo que se establezca en el Reglamento de cada Cámara, se podrán votar resoluciones, incluso fuera de los períodos de sesiones, sobre proyectos, propuestas o documentos del tipo indicado en el párrafo anterior". 
terminará la sustitución del inciso reproducido en cursivas por otro, relativo a "los proyectos de actos legislativos europeos y los demás proyectos o proposiciones de actos de la Unión Europea" ${ }^{12}$.

En cuanto al artículo 88-5 (también introducido en la reforma de 2005), la ley constitucional de julio dispone que, a partir de la entrada en vigor del Tratado de Lisboa, queda suprimida la referencia a "las Comunidades Europeas", quedando hasta entonces en los siguientes términos: "Todo proyecto de ley que autorice la ratificación de un tratado relativo a la adhesión de un Estado a la Unión Europea y a las Comunidades Europeas será sometido a referéndum por el Presidente de la República”. En todo caso, según disponen las leyes constitucionales de febrero y julio, esta disposición no será "aplicable a las adhesiones siguientes a una conferencia intergubernamental cuya convocatoria haya sido decidida por el Consejo Europeo antes del 1 de julio de 2004"13.

Finalmente, el artículo $2-4^{\circ}$ de la ley añade dos nuevos artículos (88-6 y 88-7) que, en línea con las previsiones de 2005 que no llegaron a entrar en vigor al no ratificarse el Tratado constitucional, aluden a las nuevas facultades conferidas a los Parlamentos nacionales para el control del principio de subsidiariedad y a la capacidad de oponerse a una "modificación de las reglas de adopción de actos de la Unión Europea" decidida según el procedimiento de "revisión simplificada de los Tratados o de la cooperación judicial civil".

En consecuencia, junto con el Tratado de Lisboa entrará en vigor el nuevo artículo 88-6: "La Asamblea Nacional o el Senado podrán emitir dictamen razonado sobre la conformidad de un proyecto de acto legislativo europeo con el principio de subsidiariedad. Dicho dictamen, del que se dará cuenta al Gobierno, será remitido por el presidente de la Cámara respectiva a los presidentes del Parlamento Europeo, del Consejo y de la Comisión Europea.

Cualquiera de las Cámaras podrá interponer recurso ante el Tribunal de Justicia de la Unión Europea contra actos legislativos europeos por violación del principio de subsidiariedad. El recurso será remitido por el Gobierno al Tribunal de Justicia de la Unión Europea.

Con este fin se podrán aprobar resoluciones, incluso fuera de los periodos de sesiones, según las modalidades de presentación y debate que determine el Reglamento de cada Cámara "14.

Por su parte, el 88-7 prescribirá que "Por el voto de una moción, adoptada en términos idénticos por la Asamblea Nacional y el Senado, el Parlamento podrá oponerse a la modificación de las normas de adopción de actos de la Unión Europea en los casos previstos, en materia de revisión simplificada de los tratados o de cooperación judicial civil, por el Tratado de la Unión Europea y el Tratado de Funcionamiento de la Unión Europea, modificados por el Tra-

12 Artículo 47 de la ley constitucional de 21 de julio de 2008.

13 Sobre la significación y alcance de esta disposición (modificada a su vez en julio: vid. infra, nota 38), véase nuevamente TRC, núm. 15 (2005), pág. 382.

14 Sobre la extensión de la iniciativa para presentar este recurso, vide igualmente infra, lo relativo a la reforma de 21 julio (nota 38 ). 
tado de Lisboa de 13 de diciembre de 2007”. Se establece así, para este supuesto, un procedimiento (moción aprobada por ambas Cámaras) distinto al del 88-6 (resolución de cada una de ellas). Una diferencia que, como señalamos en otro lugar, ha sido discutida, por situar al Senado en pie de igualdad con la Asamblea, desconociendo así la preeminencia constitucional de esta última. Pero cuyo sentido institucional parece claro en tanto que, al afectar a una revisión del Tratado (por más que sea simplificada), esta competencia se ejerce "en nombre del Parlamento", y no — como la anterior- por cada una de las Cámaras ${ }^{15}$.

\section{La MODERNIZACIÓN DE LAS INSTITUCIONES DE LA V REPÚBliCA}

A) El contexto

De cualquier forma, y como ya se ha apuntado al inicio de estas páginas, no cabe duda de que el debate constitucional de mayor calado que ha tenido lugar en Francia en los últimos años es el que ha rodeado a la que finalmente se ha convertido en «ley constitucional de modernización de las instituciones de la V República" ${ }^{16}$.

Dicho proyecto, como también se indicó en nuestra crónica anterior ${ }^{17}$, responde en última instancia al cumplimiento del programa electoral presentado por el actual Presidente de la República, y se concretó inicialmente en la constitución de una "Comisión de expertos sobre la modernización y el reequilibrio de las instituciones de la V República "18. La Comisión, presidida por el antiguo Primer Ministro Edouard Balladur hizo públicas sus conclusiones (plasmadas en 77 propuestas) el 29 de octubre de $2007^{19}$. A partir de ahí, "el Primer Ministro, a instancias del Presidente de la República, sometió la mayor parte de sus propuestas a un proceso de consultas con las diferentes fuerzas políticas del país, con vistas a la elaboración de un texto susceptible de recibir un amplio acuerdo" ${ }^{20}$.

Ese proceso desembocó en la aprobación de un "proyecto de ley constitucional" que fue presentado el día 23 de abril de 2008. En los términos utili-

15 TRC, núm. 15, cit.

16 Loi constitutionnelle n. ${ }^{\circ} 2008-724$ du 23 juillet 2008, de modernisation des institutions de la Ve République (Journal Officiel núm. 171, de 24 de julio, págs. 11890 y ss.).

17 "Actualidad política y constitucional francesa (2006-2007)", en TRC, núm. 20 (2007), pág. 562.

18 Decreto n. ${ }^{\circ} 2007-1108$, de 18 de julio.

19 Una síntesis de las 77 propuestas, y algunas primeras valoraciones doctrinales de las mismas, pueden encontrarse en "Rapport de la Commission Balladur: libres propos croisés de PIERRE Mazeaud et Olivier SchramecK", en Revue du Droit Public, núm. 1 (2008), págs. 3 a 49.

20 Exposición de motivos del Proyecto de ley. Cabe subrayar que éste no recogió todas las propuestas, omitiendo algunas tan relevantes como las relativas a la prohibición de la acumulación del mandato ministerial con otra función electiva, la introducción de elementos de proporcionalidad en la elección de los Diputados, la modificación del sistema de avales para los candidatos a las elecciones presidenciales, u otras. 
zados por el comunicado del Consejo de Ministros, "Cincuenta años después de la fundación de la V República, el texto propone, por primera vez, una reforma global [d'ensemble] de nuestras instituciones, dirigido principalmente a reforzar el papel del Parlamento, a renovar el modo de ejercicio del Poder Ejecutivo y a garantizar a los ciudadanos nuevos derechos. Pretende hacer posible el establecimiento de una democracia más equilibrada, sin poner en causa los caracteres esenciales de la V República".

Se trata, pues, y al menos en principio, de una reforma de carácter estructural, que como ya se ha apuntado es probablemente la más importante desde la aprobación de la elección del Presidente de la República por sufragio universal, en 1962. Por expresarlo en los términos empleados por Dominique Rousseau, «en 1958, el objetivo [constitucional] era el de poner fin a la omnipotencia del Parlamento y restaurar la autoridad del Ejecutivo. Pero los constituyentes dudaban entre atribuir el poder al Primer Ministro (tesis de Michel Debré) o al Presidente de la República (tesis del General [De Gaulle]). Resultado: se lo atribuyeron a ambos, instalando así una diarquía en la cumbre del Estado. De ahí los múltiples conflictos en el seno del Ejecutivo: entre De Gaulle y Pompidou, Pompidou y Chaban, Giscard y Chirac, Miterrand y Rocard y, actualmente, entre Sarkozy y Fillon. En 2000, el establecimiento del quinquenio [del mandato presidencial] y la inversión del calendario electoral comenzaron a zanjar esta ambigüedad fundacional: elegidos bajo el paraguas [au nom] del Presidente y a rebufo de su elección, los diputados quedan sometidos a un lazo directo y de subordinación al jefe del Estado. La figura del Primer Ministro ha quedado eliminada [est zappée], ${ }^{21}$.

Sin embargo (y como también ocurriese en 1962, salvando las muchas distancias existentes), pese a su alcance, esta reforma dista mucho de ser pacífica. Hasta el punto de que, tras ser adoptada en idénticos términos por ambas Cámaras, en segunda lectura, su aprobación definitiva en la sesión del Congreso convocada el 21 de julio dependió tan sólo de un único voto ${ }^{22}$.

En efecto, pese a que en la Comisión de expertos participaron personalidades vinculadas al Partido Socialista o próximas al mismo como Jack Lang, Olivier Duhamel o Guy Carcassone; y a pesar también del indicado proceso de consultas, lo cierto es que el proyecto no ha conseguido un respaldo unánime: a grandes rasgos, ha sido muy mayoritariamente —al final, casi tajantemente- rechazado por los principales partidos de la oposición de izquier-

21 VIDE RAULIN, NATHALIE, : Institutions. Le constitutionnaliste Dominique Rousseau sur le projet de réforme", en Libération, 15 de julio de 2008. Sobre las reformas citadas, puede verse las crónicas incluidas en TRC, números 5 y 8 (2000 y 2001, págs. 239-243 y 288-290, respectivamente).

22 El texto fue aprobado en primera lectura por la Asamblea Nacional el día 3 de junio, por 315 votos contra 231; y por el Senado, con modificaciones, el 24 de junio, por 166 votos a favor y 123 en contra. En segunda lectura, la Asamblea aprobó el texto el día 9 de julio, y el Senado en términos idénticos- el día 16 (con 162 votos favorables y 125 en contra). Finalmente, el Parlement reuni en Congrès aprobó la reforma por 539 votos contra 357, siendo la mayoría requerida de 538 votos. 
das $^{23}$. Por su parte, los Grupos centristas han oscilado entre el apoyo crítico y la abstención, al margen de algún voto negativo ${ }^{24}$; y en las propias filas del partido mayoritario, la UMP, se han registrado algunas defecciones ${ }^{25}$.

De cualquier manera, y dado que el cierre de estas páginas se ha retrasado para recoger el resultado final de la votación, parece necesario describir, aunque sea de forma muy somera, los principales contenidos de la reforma, así como los argumentos que han protagonizado el debate en torno a la misma. Porque, en definitiva, lo que está en juego es la capacidad de adaptación de una Constitución que en octubre de este año celebrará su 50 aniversario, y que ha sido objeto de reformas parciales, pero a veces profundas, como las ya citadas relativas al modo de elección del Presidente de la República o a la duración de su mandato.

\section{B) El texto}

La dimensión de la reforma finalmente aprobada resulta patente atendiendo simplemente a un criterio cuantitativo: afecta a una cincuentena de artículos, más de la mitad de los que tiene el texto constitucional vigente ${ }^{26}$.

No obstante, su alcance queda más claro cuando se observan las concretas innovaciones incorporadas al texto constitucional, cuya eficacia sólo podrá, obviamente, evaluarse con el transcurso del tiempo, y que ahora sólo podemos describir muy superficialmente. De cualquier forma, y de acuerdo con el planteamiento expresado en la Exposición de motivos que acompañó al proyecto de ley, dichas innovaciones se articulan en torno a tres grandes lí-

23 En la primera lectura en la Asamblea, 190 Diputados del "Grupo Socialista, Radical, Ciudadano y de independientes de izquierda [divers gauche]" votaron contra el texto, 1 a favor y 10 se abstuvieron. En la votación final del Congreso, 194 diputados y 95 senadores socialistas votaron en contra, pero la mayoría de los parlamentarios del Partido de los Radicales de Izquierda (PRG, tradicional aliado de los socialistas) votaron a favor de la reforma; al igual que hizo el propio Jack Lang, diputado socialista por la circunscripción de Pas-de-Calais. Más a la izquierda, la oposición ha sido aún mayor: los 24 diputados del Grupo "Izquierda Democrática y Republicana", al igual que los 23 senadores del Grupo "Comunista, Republicano y Ciudadano" se pronunciaron sistemáticamente, y hasta la votación final, contra el texto.

24 Finalmente, de los 24 diputados del "Nuevo Centro" [Nowveau Centre], 23 apoyaron la reforma y otro se abstuvo; de los 30 senadores de "Unión Centrista-UDF", 24 optaron por el "Sí", dos por el "no" y 4 se abstuvieron. Entre los diputados "no inscritos", votaron que no tres diputados del también centrista Mouvement Démocratique (MoDem), entre ellos su líder F. Bayrou.

25 Así, en las sucesivas votaciones en la Asamblea y el Senado se abstuvieron o votaron en contra del proyecto algunos representantes de la mayoritaria UMP, entre ellos figuras de relieve como los ex Ministros Hervé de Charette, Charles Pasqua o Bernard Debré. En la votación final en el Congreso, y tras una intensa presión por parte de los dirigentes de la mayoría, la práctica totalidad de los miembros de la UMP (310 diputados y 158 senadores, entre ellos los tres anteriormente citados) votaron favorablemente, aunque seis diputados y un senador lo hicieron en contra, y otro más se abstuvo.

26 El último artículo del texto vigente es el 89; sin embargo, las diversas reformas anteriores han creado otros 18 artículos (cuya numeración adopta la fórmula que ya se ha visto antes en materia de adaptación a la Unión Europea: 88-1, 88-2, 88-3, etc.; y que ahora vuelve a utilizarse en la creación de otros nuevos: 34-1, 47-2, 51-1 y 51-2, 61-1 y 71-1); vaciando de contenido otros once (en concreto, los arts. 75 y 78 a 87 ). 
neas, que se complementan recíprocamente: «un poder ejecutivo mejor controlado, un Parlamento profundamente reforzado y nuevos derechos para los ciudadanos".

En las líneas que siguen, trataremos de resumir las principales novedades que aporta la reforma. A tal fin, y asumiendo de antemano que muchas de ellas difícilmente pueden ser examinadas desde una única perspectiva, intentaremos agruparlas para examinar, primero, las medidas dirigidas a reforzar los derechos y libertades de los ciudadanos (a); a continuación, a las que modifican las relaciones existentes entre Parlamento y Ejecutivo (b); para finalizar con una breve referencia a otras innovaciones, más heterogéneas, que afectan a diversas instituciones y modifican otros preceptos constitucionales (c).

\section{a) Las nuevas garantias de los derechos y libertades}

Este aspecto, igualmente latente en muchas disposiciones que veremos al desarrollar el nuevo equilibrio entre Parlamento y Ejecutivo, se aprecia con toda nitidez en las siguientes disposiciones ${ }^{27}$ :

(i) Indiscutiblemente, una de las medidas estelares, más innovadoras y menos polémicas de la reforma es la aceptación del control de constitucionalidad de las leyes por vía de excepción (lo que podríamos denominar, con muchos matices, cuestión de inconstitucionalidad) en esta materia. En efecto, de acuerdo con el nuevo artículo 61-1 de la Constitución, "cuando, con ocasión de un procedimiento judicial en curso, se considere que una disposición legislativa vulnera los derechos y libertades garantizados por la Constitución, la cuestión podrá ser planteada ante el Consejo Constitucional por el Consejo de Estado o por la Corte de Casación" (Tribunal Supremo), en los términos que establezca una ley orgánica ${ }^{28}$.

La admisión de esta figura, que — en los términos del Comunicado del Consejo de Ministros que presentó el proyecto de ley- "permitirá a todo justiciable discutir la conformidad de una disposición legislativa a los derechos y libertades reconocidos en la Constitución en cualquier instancia en la que sea parte" obliga a modificar también el artículo 62, relativo a los efectos de las decisiones del Consejo Constitucional que declaran la inconstitucionalidad de una disposición con rango de ley. De esta manera, «una disposición declarada inconstitucional" por esta vía de excepción "será derogada a partir de la publicación de la decisión del Consejo Constitucional, o de una fecha posterior fijada por dicha decisión. El Consejo determinará las condiciones y los límites en que los efectos anteriormente producidos por la disposición afectada pueden ser cuestionados".

27 La ley constitucional cuenta con 47 artículos pero, al objeto de no complicar más de lo necesario la exposición, las modificaciones se citarán fundamentalmente atendiendo a su ubicación en el texto constitucional. Ello no obstante, cuando resulte conveniente, se citará el artículo concreto de la ley constitucional en que la reforma se incluye. En otro orden de cosas, la traducción de los textos se debe al autor de esta crónica, a quien son pues imputables todos los errores que puedan apreciarse.

28 Las cursivas han sido añadidas, subrayando el concreto ámbito material de esta figura. 
(ii) Seguramente, otra de las principales novedades propuestas es la creación de la figura del "Defensor de los Derechos" [Défenseur des droits]. A tal fin, se crea un nuevo Título XI-bis, formado por un único artículo (71-1) según el cual su misión es la de "velar por el respeto de los derechos y libertades por parte de las Administraciones [públicas]..., así como de todo organismo investido de una misión de servicio público o respecto del cual sea competente, según su ley orgánica", encargada de regular sus atribuciones y modalidades de actuación.

Estará legitimada para acudir al Defensor "cualquier persona que se estime lesionada por el funcionamiento de un servicio público" o de cualquier organismo incluido en el apartado anterior, pudiendo actuar igualmente de oficio. En cuanto a su nombramiento, corresponderá al "Presidente de la República para un mandato no renovable de seis años", debiendo rendir cuenta de su actividad tanto al Presidente como al Parlamento.

(iii) En el artículo 11 de la Constitución (al que luego volveremos) se incluyen cuatro nuevos párrafos $\left(3^{\circ}\right.$ a $\left.6^{\circ}\right)$ que introducen y fijan las reglas básicas de funcionamiento de un instituto completamente novedoso, como es un referéndum a iniciativa, compartida, popular y parlamentaria. Así, "Podrá organizarse un referéndum sobre alguno de los objetos señalados en el párrafo primero a iniciativa de una quinta parte de los miembros del Parlamento, respaldada por una décima parte de los electores inscritos en el censo electoral. Tal iniciativa adoptará la forma de proposición de ley, y no podrá tener por objeto la derogación de una disposición legislativa promulgada desde hace menos de un año", remitiéndose a una ley orgánica las condiciones de su presentación y de su control por parte del Consejo Constitucional (que será preceptivo, según señala la nueva redacción del artículo 61, párrafo primero).

Se trata, ciertamente, de una figura cuya puesta en práctica resultará difícil (en especial por el alto número de ciudadanos que deben impulsarla), pero que, desde luego, no parece imposible.

(iv) El principio de igual acceso de mujeres y hombres a los mandatos electorales y funciones electivas", introducido en el artículo 3 por la ley constitucional 99-569, de 8 de julio ${ }^{29}$, extiende su alcance hasta abarcar "las responsabilidades profesionales y sociales", y pasa a ubicarse como segundo párrafo del artículo $1^{30}$.

29 Cfr. TRC, núm. 3 (1999), págs. 242 a 244.

30 Esta innovación, que consagra la llamada "paridad profesional", resulta de una enmienda presentada por varios diputados de la mayoritaria UMP ("enmienda Zimmermann") contra la opinión gubernamental, pese a lo cual fue aprobada por la Asamblea Nacional, y posteriormente mantenida en el Senado. Su objetivo es el de salvar la objeción planteada por el Consejo Constitucional frente a las disposiciones relativas al acceso de mujeres a diversas instancias profesionales y jurisdiccionales (consejos de administración, comités de empresa, etc.) que, a juicio del Alto Tribunal, no estaban cubiertas por el principio de igual acceso de hombres y mujeres introducido en el artículo 3 de la Constitución, cuyo ámbito de aplicación se limitaba "a las elecciones a mandatos y funciones políticas" (Décision 2006-533 DC, de 16 de marzo de 2006, especialmente Considerandos 11 a 16). 
(v) Dentro ya del Título I (De la soberanía"), se incorpora al artículo 4 (relativo a los partidos políticos) un segundo párrafo según el cual «la ley garantiza las expresiones pluralistas de las opiniones y la participación equitativa [équitable] de los partidos y grupos políticos en la vida democrática de la Nación". Un principio que encuentra diversas concreciones en otros puntos de la reforma.

Así, un nuevo artículo dispone que «El Reglamento de cada Cámara determina los derechos de los Grupos Parlamentarios constituidos en su seno, reconociendo derechos específicos a los Grupos de oposición y a los Grupos minoritarios" (artículo 51-1).

De otra parte, ya en el Título V ("De las relaciones entre el Parlamento y el Gobierno") la protección del pluralismo social y de la libertad de expresión se ve reforzada mediante la ampliación del ámbito material reservado a la ley, que se extiende a "la libertad, el pluralismo y la independencia de los medios de comunicación" [des médias] (artículo 34).

(vi) En todo caso, otras modificaciones inciden también sobre los derechos de los ciudadanos, aunque no sea ése su objetivo directo. Así, la reforma del Consejo Superior de la Magistratura abre la puerta a que "un justiciable" pueda acudir [saisir] al mismo "en las condiciones fijadas por una ley orgánica" (artículo 65); mientras que la del Consejo Económico y Social (que pasa a denominarse "Económico, Social y Medioambiental [environnemental]": artículos 69 a 71 de la Constitución) posibilita igualmente el acceso al mismo "por vía de petición en las condiciones fijadas por una ley orgánica", prescribiéndose que «tras examinar la petición, comunicará al Gobierno y al Parlamento" sus propuestas de actuación.

En este mismo apartado podrían incluirse otras disposiciones, como aquella —una de las pocas que ha alcanzado algún eco en España, al hilo de nuestros propios debates sociales y constitucionales - que dispone que «las lenguas regionales pertenecen al patrimonio de Francia" (artículo 75-1), o la que plasma la voluntad de "la República" de "participa[r] en el desarrollo de la solidaridad y la cooperación entre los Estados y los pueblos que tienen en común el francés ${ }^{31}$.

\section{b) La modificación de la relación entre Parlamento y Ejecutivo}

La mayor parte de la reforma se dirige directamente a este objetivo. Y, en línea con el análisis que hacía Dominique Rousseau, lo hace modificando, de una parte, la posición del Presidente de la República y su relación con el Parlamento (b.1); y, de otra, la relación entre el Parlamento y el Gobierno (b.2). En ambos casos, como trataremos de exponer, se refuerzan las funciones del órgano representativo, respondiendo así a una cuestión ya clásica en el sistema francés ${ }^{32}$.

31 "Ayant le français en partage" (art. 87). Consecuentemente, el Título XIV de la Constitución ("De los acuerdos de asociación") pasa a denominarse "De la francofonía y de los acuerdos de asociación".

32 Vid. CHANDERNAGOR, ANDRÉ: Un Parlement, pour quoi faire? Paris, Gallimard, 1967. 


\section{b.1) El Presidente de la República, y su relación con el Parlamento}

(i) En este apartado, cabe destacar en primer lugar la limitación de los mandatos presidenciales. En efecto, en el artículo 6 se introduce un segundo párrafo según el cual "nadie podrá ejercer más de dos mandatos consecutivos" en la Presidencia de la República. Una medida que no aparecía en la propuesta de la "Comisión Balladur", pero fue incorporada, al parecer, a instancias del propio Presidente Sarkozy.

(ii) Ahora bien: la medida que más atención ha despertado en este ámbito es, sin duda, la que dispone que el Presidente de la República "podrá tomar la palabra ante el Parlamento, reunido a tal efecto en Congreso. Su declaración podrá dar lugar, sin su presencia, a un debate, que no podrá desembocar en ninguna votación "33. Se pone así fin a una prohibición vigente desde la III República, y que convertía a la tribuna parlamentaria en el lugar que simboliza la responsabilidad política del Primer Ministro y de su Gobierno.

En ese contexto, esta propuesta ha sido objeto de duras críticas no sólo por parte de la izquierda política, que la considera un paso más en el proceso de personalización del régimen político francés, sino también de destacados representantes del gaullismo tradicional ${ }^{34}$.

(iii) Tampoco cabe ignorar la importancia de que el poder de nombramiento del Presidente de la República — hasta ahora libre, más allá de la exigencia de que en algunos casos se produzca en Consejo de Ministros- se someta al examen parlamentario. Algo que se deriva de la enmienda que ha incorporado al artículo 13 de la Constitución un quinto párrafo, en cuya virtud gran parte de los nombramientos atribuidos al Presidente de la República, «en razón de su importancia para la garantía de los derechos y libertades o para la vida económica y social de la Nación" sólo podrán llevarse a cabo "tras informe [avis] público de la Comisión permanente competente de cada Cámara". Es más: «El Presidente de la República no podrá proceder a un nombramiento cuando la suma de votos negativos en cada Comisión represente como mínimo tres quintos de los votos emitidos". En definitiva, se concede a las Comisiones parlamentarias competentes (que serán determinadas por la ley "según los cargos o funciones afectados") un auténtico "poder de veto" frente a los candidatos presidenciales.

33 El proyecto inicial preveía que el Presidente pudiera dirigirse a la Asamblea Nacional, al Senado o a ambas Cámaras conjuntamente. Sin embargo, la Cámara Baja limitó esta posibilidad.

34 Desde la izquierda, se ha resaltado que esta medida contribuye a debilitar al Primer Ministro. Así, el Senador y antiguo ministro socialista Robert Badinter señalaba que "el público verá físicamente al Presidente de la República como líder de la mayoría. Y en tal caso, ¿een qué se convierte el Primer Ministro, silencioso y escondido [tout petit] en su escaño entre los ministros?" (citado por RAULIN, NATALIE, "Une réforme qui affaiblit le Premier Ministre", en Libération, 17 de julio de 2008). Desde el otro lado del espectro político, pueden mencionarse las palabras de Bernard Debré, hijo de Michel Debré y diputado de la mayoritaria UMP: «viene a depreciar la función presidencial porque el Jefe del Estado, al venir al hemiciclo, se hará abuchear [huer] por la oposición, quienquiera que sea el Presidente, cualquiera que sea la oposición" (citado por www.lefigaro.fr, 20 de mayo de 2008). 
Esta previsión se aplicará incluso al nombramiento de los miembros del Consejo Constitucional, con la salvedad de que los designados por los Presidentes de ambas Cámaras «estarán sometidos únicamente al informe de la Comisión permanente competente de la Cámara afectada" (inciso añadido al primer párrafo del artículo 56).

(iv) En la misma línea de reforzar el control parlamentario de la acción del ejecutivo, se establece un poderoso límite adicional a los poderes excepcionales conferidos al Presidente de la República por el artículo 16 cuando «las instituciones de la República, la independencia de la Nación, la integridad de su territorio o la ejecución de sus compromisos internacionales sean amenazados de manera grave e inmediata". En efecto, "Tras treinta días de ejercicio de los poderes excepcionales, el Presidente de la Asamblea Nacional, el Presidente del Senado, sesenta Diputados o sesenta Senadores podrán acudir al Consejo Constitucional, a fin de examinar si se reúnen las condiciones exigidas en el primer párrafo [del artículo]. El Consejo se pronunciará en el plazo más breve por un informe público [avis public]. Transcurrido un plazo de sesenta días de ejercicio de los poderes excepcionales, y en cualquier momento más allá de ese término, el Consejo procederá a este examen de oficio y se pronunciará en las mismas condiciones".

(v) Otro poder característico del Presidente de la República, como es el ejercicio del derecho de gracia, se ve también restringido, al incluirse en el artículo 17 un último inciso que lo limita "a título individual", excluyendo pues los «indultos generales".

(vi) Aunque su relevancia sea menor, también puede mencionarse la ampliación de la lista de materias susceptibles de ser sometidas a referéndum por iniciativa del Presidente (y desde ahora, como ya se ha visto, de parlamentarios y ciudadanos): a las «leyes relativas a la política económica o social" se añaden las de carácter "medioambiental" (artículo 11).

\section{b.2) Relaciones entre el Parlamento y el Gobierno}

(i) Con carácter general, cabe señalar que, conforme a la nueva redacción dada al artículo 24, el Parlamento suma a su tradicional función legislativa (que deja de estar definida en el artículo 34) las de "control de la acción del Gobierno" y "evaluación de las políticas públicas”.

Esa nueva orientación inspira muchas medidas concretas. Así,

- El Tribunal de Cuentas [Cour des comptes] «asiste al Parlamento en el control de la acción del Gobierno. Asistirá igualmente al Parlamento y al Gobierno en el control de la ejecución de las leyes presupuestarias... así como en la evaluación de las políticas públicas". Añadiendo, además, que "mediante sus informes públicos, contribuye a la información de los ciudadanos" (artículo 47-2, de nueva creación).

- En términos similares, se amplía al Parlamento la capacidad de solicitar informes "sobre cualquier problema de carácter económico, social o medioambiental" al Consejo Económico, Social y Medioambiental (artículo 70$)$. 
- Asimismo, "para el ejercicio de sus funciones de control y evaluación definidas en el... artículo 24, podrán crearse Comisiones de Encuesta [d'enquête] en el seno de cada una de las Cámaras para reunir elementos de información, en las condiciones previstas por la ley" (artículo 51-2).

- De otra parte, se prevén «leyes de programación [que] determinan los objetivos de la acción del Estado", definen "las orientaciones plurianuales de las finanzas públicas" y "se inscriben en el objetivo de equilibrio presupuestario de las administraciones públicas", objetivo que adquiere así rango constitucional (artículo 34).

- A continuación, se atribuye a las Cámaras el poder (hasta ahora inexistente) de "Votar resoluciones, en las condiciones fijadas por la ley orgánica". Con la salvedad de que "son inadmisibles y no pueden ser inscritas en el orden del día las propuestas de resolución cuya adopción o rechazo, a juicio del Gobierno, pudiese por su naturaleza poner en juego su responsabilidad, (nuevo artículo 34-1). En el ámbito comunitario, la nueva redacción del artículo 88-4 (que deja sin efecto, pues, la resultante de la reforma de febrero) prevé la adopción de "resoluciones europeas".

- Otra novedad del texto es la previsión de que se produzcan "debates de actualidad", de forma que "el Gobierno puede, a iniciativa propia o a petición de un Grupo parlamentario..., hacer una declaración sobre un asunto determinado ante cualquiera de las Cámaras, dando lugar a un debate. Puede asimismo, si así lo decide, someterla a votación sin comprometer su responsabilidad" (artículo 50-1).

- Particularmente llamativa resulta la extensión del control parlamentario a la actividad de las Fuerzas Armadas fuera de Francia. Así, al artículo 35 (que exigía la autorización parlamentaria de cualquier "declaración de guerra") se añaden tres párrafos, de acuerdo con los cuales «el Gobierno informa al Parlamento de su decisión de hacer intervenir las Fuerzas Armadas en el extranjero, como máximo tres días después del inicio de la intervención, precisando los objetivos perseguidos. Esta información podrá dar lugar a un debate, sin votación". Ahora bien: "Cuando la duración de la intervención exceda de cuatro meses, el Gobierno someterá su prolongación a la autorización del Parlamento", decidiendo en última instancia la Asamblea Nacional. Y ello, aun cuando el Parlamento no se encuentre reunido al expirar el período de cuatro meses, ya que en tal caso "se pronunciará al iniciarse el siguiente período de sesiones".

(ii) Por otro lado, la reforma de los procedimientos parlamentarios incide directa y profundamente en la posición que en ellos ocupan el Parlamento y el Gobierno.

- Una de las reformas más relevantes, por afectar a uno de los procedimientos parlamentarios más característicos —y polémicos- de la V República, es la que ha reducido drásticamente el alcance del artículo 49, párrafo 3 de la Constitución. Un mecanismo que permite al "Primer Ministro, previa deliberación del Consejo de Ministros,... comprometer la responsabilidad del Gobierno ante la Asamblea Nacional en relación con la votación de un texto. 
En tal caso, el texto se considerará aprobado", salvo que se adopte una moción de censura, presentada en las veinticuatro horas siguientes. Y cuya eficacia política para cohesionar a la mayoría en torno al Gobierno ha sido suficientemente probada por la experiencia de la V República ${ }^{35}$.

Pues bien: de acuerdo con la nueva redacción de este párrafo, tal posibilidad subsiste, pero queda limitada a los proyectos de ley de presupuestos del Estado y de financiación de la Seguridad Social. Fuera de esos dos casos, el Primer Ministro sólo podrá "recurrir a este procedimiento en relación con otro proyecto u otra proposición de ley por período de sesiones, ${ }^{36}$.

- En otro orden de cosas, se consagra en la Constitución la obligación de que las ordenanzas gubernamentales (similares, en algún sentido, a nuestros Decretos legislativos) deban ser expresamente ratificadas por las Cámaras (artículo 38).

- Igualmente importante es la reforma de las normas que regulan la formación del orden del día de las Cámaras. Hasta la fecha, según el artículo 48, dicho "orden del día... comprenderá, prioritariamente y en el orden fijado por el Gobierno, la discusión de los proyectos de ley presentados por el Gobierno y las proposiciones de ley aceptadas por él", por más que "al menos una sesión por semana estará reservada prioritariamente a las preguntas de los miembros del Parlamento y a las respuestas del Gobierno", y "una sesión por mes se reserve prioritariamente al orden del día fijado por cada Cámara".

Resultan evidentes las limitaciones que esta ordenación implicaba para toda iniciativa parlamentaria. Y, en consecuencia, también el alcance de la nueva redacción de este artículo, que modifica el punto de partida al señalar que "el orden del día es fijado por cada Cámara".

A partir de ahí, ciertamente, "dos semanas de sesiones de cada cuatro se reservan prioritariamente, y en el orden fijado por el Gobierno, al examen de los textos y a los debates que el mismo solicite que se inscriban en el orden del dían. Y se mantiene la prioridad otorgada a ciertos proyectos de ley (presupuestos del Estado o de la Seguridad Social, situaciones de crisis, etc.). Pero no es menos cierto que "una semana de cada cuatro queda reservada prioritariamente y en el orden fijado por cada una de las Cámaras al control de la acción del Gobierno y a la evaluación de las políticas públicas", añadiéndose que "un día al mes se reserva a un orden del día decidido por cada Cámara" a iniciativa de los grupos de oposición y minoritarios de la misma. Finalmente,

35 Así, este instrumento ("el recurso a la Gran Berta", según JEan GICQUEL, Droit consti-

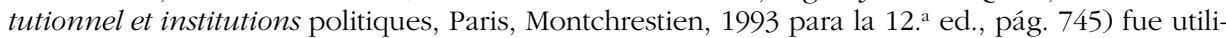
zado 76 veces entre 1959 y 1995, en lo que —otra vez — se ha considerado una "banalización" de un procedimiento inicialmente concebido como excepcional (vide Favoreu, Lous et al., Droit Constitutionnel. Paris, Dalloz, 1999 para la 2. ${ }^{\text {e }}$ ed., pág. 697). Como se ha recordado durante el reciente debate, su uso permitió por ejemplo la creación de un arsenal nuclear francés (la force de frappe); pero no fue ni mucho menos exclusivo del gaullismo: así, Michel Rocard lo utilizó en 24 ocasiones durante los tres años en que fue Primer Ministro (1998-2001) a fin de forzar la disciplina de la mayoría de la izquierda (PSF-PCF) que lo sostenía, no siempre homogénea.

36 De octubre a junio (artículo 28 C.). 
se mantiene la sesión semanal de control, incluyendo durante los períodos de sesiones extraordinarios.

- Aunque menos llamativa, también resulta extraordinariamente relevante la reforma del artículo 42 de la Constitución. De una parte, porque hasta el momento "la discusión [plenaria] de los proyectos de ley" en la Cámara a la que fueran inicialmente sometidos tenía por objeto «el texto presentado por el Gobierno", y no el resultante de su estudio en Comisión. Desde ahora (y con algunas salvedades: proyectos de ley presupuestarios o de reforma constitucional), la discusión versará sobre el texto adoptado en Comisión, de acuerdo con la lógica parlamentaria.

De otra parte, porque el mismo artículo (completado, para las leyes orgánicas, por el artículo 46; y para los proyectos de reforma constitucional, por el 89) introduce unos plazos mínimos para que los proyectos o proposiciones de ley (con algunas excepciones, igualmente tasadas) puedan ser discutidos por los Plenos de las Cámaras, en primera lectura. Así, la Cámara ante la que se presente el texto no puede examinarlo sin que transcurran seis semanas desde su presentación (quince días, cuando se trate de leyes orgánicas respecto de las cuales el Gobierno haya declarado la urgencia); mientras que, cuando el texto pase a la otra Cámara, ésta también deberá respetar un plazo mínimo de cuatro semanas.

- Muchos otros aspectos han sido objeto de diversas y significativas modificaciones. Entre ellos, cabe citar el derecho de enmienda, que se amplía (artículos 44 y 45, párrafo primero). Así como la declaración de urgencia por parte del Gobierno (que podrá ser contestada por las Juntas de Portavoces [Conférences des Présidents] de ambas Cámaras, conjuntamente: art. 45, párrafo segundo). O la presentación de proyectos y proposiciones de ley (artículo 39, que introduce un procedimiento por el cual se atribuye al Consejo Constitucional el enjuiciamiento sobre el cumplimiento de los requisitos legalmente exigidos para los proyectos, en caso de discrepancia entre Gobierno y Junta de Portavoces). También se atribuye a los Presidentes de las Cámaras la capacidad (hasta ahora exclusiva del Gobierno) para oponerse a la tramitación de proposiciones o enmiendas que excedan del dominio de la ley, o se opongan a delegaciones legislativas en vigor (artículo 41). Además, se reforman las reglas relativas al examen en Comisión de los proyectos o proposiciones de ley (artículo 43)... Finalmente, debe mencionarse aquí la creación, en ambas Cámaras, de sendas Comisiones de Asuntos Europeos, de acuerdo con la nueva redacción del artículo 88-4.

\section{c) Otras innovaciones}

Este apartado, mucho más heterogéneo, debe iniciarse con la reforma que, rompiendo con una de las características más peculiares de la "separación de poderes" en su interpretación de la V República, permite a los parlamentarios volver a ocupar su escaño tras ocupar cargos en el ejecutivo, sin necesidad de someterse, como ocurría hasta ahora, a una elección parcial. De 
este modo, el artículo 25 admite «la sustitución temporal" de los parlamentarios en tal caso, volviendo a la práctica usual en la III y IV Repúblicas.

El mismo artículo prevé la creación de "una Comisión independiente", cuya composición y reglas de funcionamiento serán fijados por la ley, encargada de pronunciarse "mediante informe público", sobre cualquier propuesta legislativa "que delimite las circunscripciones para la elección de los diputados o modifique la distribución de escaños de diputados o senadores".

En otro orden de cosas, los artículos 24 y 71 incorporan la fijación de un número máximo de diputados (577), senadores (348) y de miembros del Consejo Económico, Social y Medioambiental (233). Igualmente, se introduce el principio de que los franceses residentes en el extranjero están representados no sólo en el Senado, como hasta ahora, sino también en la Asamblea Nacional, en los términos que fije la ley (artículos 24 y 34, párrafo octavo).

Al margen de otros aspectos ya señalados relativos al Consejo Constitucional, éste queda también encargado de examinar, con carácter previo y preceptivo, el texto de las proposiciones de ley que, según lo dispuesto en el reformado artículo 11, hayan de ser sometidos a referéndum (artículo 61, párrafo primero).

En cuanto al Consejo Superior de la Magistratura, sufre también una importante reforma, que incide tanto sobre su composición como sobre su funcionamiento. Desde el primer punto de vista, deja de estar presidido por el Presidente de la República y, en su defecto, por el Ministro de Justicia, pasando la presidencia al Primer Presidente de la Corte de Casación y al Procurador General ante la misma (dada la existencia de dos formaciones, una relativa a los Jueces y Magistrados, y otra a los Fiscales). Además, los Magistrados o Fiscales dejan de ser mayoría, frente a otros componentes de cada una de esas formaciones (un Consejero de Estado designado por el Consejo, un abogado, y seis personalidades no pertenecientes al Parlamento, a la judicatura ni a la jurisdicción administrativa, designadas, previa comparecencia parlamentaria, por el Presidente de la República y los Presidentes de cada una de las Cámaras).

De otra parte, se modifican también las reglas relativas a su funcionamiento como órgano disciplinario y consultivo del Presidente de la República en materia de justicia, incorporándose — según se señaló anteriormente- la posibilidad de que cualquier justiciable pueda acudir al mismo.

Para finalizar este repaso, y dejando al margen algunos otros cambios relativos a los territorios de Ultramar ${ }^{37}$, cabe tan sólo mencionar dos innovaciones de indiscutible interés en materia de integración europea, que vienen a completar el régimen establecido por la reforma constitucional de febrero de este mismo año ${ }^{38}$.

El primero de ellos afecta al artículo 88-5, que - como es sabido- prevé que cualquier ampliación de la Unión sea sometida a referéndum. Pues bien:

37 Vide artículos 72-3, 73 y 74-1 C.

38 Vid. supra, notas números 13 y 14. 
el debate sobre este precepto (y, muy particularmente, sobre su aplicación al caso de Turquía) ha desembocado en un punto de acuerdo, conforme al cual "el Parlamento, mediante el voto de una moción adoptada en términos idénticos por una mayoría de tres quintos en cada una de las Cámaras, podrá autorizar la adopción del proyecto de ley [que autorice la ratificación del tratado relativo a la adhesión de un nuevo Estado a la Unión] según el procedimiento previsto", para la reforma constitucional, en el párrafo tercero del artículo 89. En otras palabras: si las Cámaras así lo deciden, el referéndum podrá ser sustituido por la aprobación del Parlamento, reunido en Congrès, por una mayoría cualificada de tres quintos.

El segundo, no menos llamativo, es el que otorga a sesenta diputados o sesenta senadores (y, por tanto, a las minorías) la iniciativa para que la Cámara respectiva formalice un recurso ante el Tribunal de Justicia de las Comunidades Europeas contra un acto legislativo comunitario, por violación del principio de subsidiariedad (artículo 88-6).

C) El debate

Para acabar estas páginas, es preciso señalar que el debate y la aprobación final de esta "modernización" de las instituciones ha estado rodeado, e influido, por la discusión acerca de otros aspectos no incluidos en la misma, pero que han sido relevantes a la hora de determinar la posición de las diferentes fuerzas políticas. Porque, como ya se ha apuntado, las principales formaciones de la izquierda política y parlamentaria eran en principio hostiles a una reforma que contemplaban como un paso más en la "presidencialización" del régimen ${ }^{39}$; y otros sectores (centristas, gaullistas) tenían otras varias reservas.

En este sentido, no deja de ser significativo que, según han subrayado los medios franceses (y por más que la afirmación sea más que matizable), el voto que ha permitido aprobar la reforma, contra la posición casi unánime de su partido, haya sido el de Jack Lang, diputado y ex Ministro socialista que, como se recordará, fue incluido ya en la Comisión Balladur que está en el origen de la reforma. En definitiva, la estrategia inicial de superar los alineamientos partidistas se ha mostrado, aunque sea por muy poco, como eficaz. Aunque, evidentemente, si no ha ido más allá se ha debido a otros varios argumentos.

39 Es más: en una línea crítica que puede resultar sorprendente en el caso de los socialistas (no así en el de los comunistas), el portavoz socialista en la Asamblea, Arnaud Montebourg, señalaba que "hay que abrir las puertas y ventanas de la V República... en una palabra, avanzar hacia un sistema político diferente, con otras reglas de representación, es decir, otras fórmulas electorales" (intervención en el turno de explicación de voto, durante la segunda lectura del proyecto ante la Cámara Baja, el 9 de julio de 2008). Por su parte, el portavoz comunista, Jean-Pierre Brard, denunció en el mismo acto "la deriva monárquica del poder", considerando que "lo que bloquea la democracia en nuestro país... es el hecho mayoritario [le fait majoritaire]". A su juicio, "la V República está al borde del agotamiento", por lo cual "lo que necesitamos es una nueva Constitución". 
De esta manera, la oposición —especialmente, la socialista- manifestó, en diversos momentos de la tramitación parlamentaria de la reforma, que su voto negativo era "temporal", hablando incluso de un "no de esperanza". Y se justificaba aludiendo, fundamentalmente, a dos puntos: en primer lugar, la fórmula de elección de los senadores; en segundo término, la monopolización del espacio público por las intervenciones del Presidente de la República.

En cuanto al primero de estos aspectos, es bien sabido que el sistema de elección de los senadores ha sido muy polémico durante toda la República, registrando en la última década varias reformas dirigidas a reducir la sobrerrepresentación de los municipios menos poblados en el colegio electoral senatorial, y la consiguiente infrarrepresentación de la población concentrada en los núcleos urbanos ${ }^{40}$. Pues bien: la izquierda $-\mathrm{y}$ buena parte de los grupos centristas- consideraban esencial reflejar en la Constitución un principio que vinculase la elección de los senadores a la población de los departamentos. Algo que, finalmente, no se incluyó — pese a que en algún momento sí se contemplara $^{41}$ - , sin que fueran suficientes algunas manifestaciones del Presidente Sarkozy que, en vísperas de la definitiva sesión del Congreso, declaró estar "abierto" a la discusión sobre este punto ${ }^{42}$.

La segunda cuestión problemática es la que el portavoz socialista en la Asamblea Nacional definió como "la cuasi-monopolización del tiempo de palabra en los medios de comunicación", reforzada por "el control... de las cadenas de televisión, de las emisoras de radio y de los medios escritos" ${ }^{43}$. Tampoco a este respecto fue suficiente la "oferta" hecha, igualmente a última hora, por el Presidente, al declararse dispuesto a "garantizar a la oposición un derecho de respuesta cada vez que el Presidente haga declaraciones relativas a la política francesa " ${ }^{44}$. Una oferta cuya concreta articulación debería correr a cargo del Consejo Superior de lo Audiovisual (CSA), encargado de velar por el respeto al pluralismo social y político en los medios de comunicación, y a quien correspondería determinar qué intervenciones son "institucionales" (y no darían lugar, por tanto, a tal respuesta) y, por el contrario, cuáles son "políticas".

De cualquier forma, otras cuestiones pudieron tener incidencia a la hora de convencer a los indecisos. Es el caso, particularmente, de la posibilidad de

40 Vid. TRC, núms. 3 (1999), 5 (2000) y 14 (2004), págs. 261, 250-251 y 417-418, respectivamente.

41 Así, el proyecto inicial indicaba que "el Senado... asegura la representación de las colectividades territoriales de la República teniendo en cuenta su población", pero este último inciso desapareció durante su paso por el Senado, sin que volviese a recuperarse.

42 "Todo el mundo sabe que yo hubiese querido ir algo más lejos sobre la reforma del Senado. Eso no ha sido posible, pero hay senadores abiertos a la idea de una reforma". En particular, consideró "razonable" una proposición presentada en 1999 por un senador —actualmente, en las filas de la UMP_, dirigida a "asegurar un mejor equilibrio entre demografía y territorio mediante una representación más equitativa de las grandes aglomeraciones" (entrevista concedida a Le Monde, 17 de julio).

43 Intervención de Arnaud Montebourg, citada.

44 Entrevista a Le Monde, citada. Una proposición de ley socialista en este sentido había sido rechazada el 22 de mayo en la Asamblea Nacional. 
reducir el número de diputados exigido para formar un Grupo Parlamentario (actualmente fijado en veinte) a quince, lo que haría posible la formación de otros Grupos (en particular, el de los Radicales de Izquierda, PRG). En la misma entrevista, Sarkozy se mostró favorable a esta medida, que en todo caso debería adoptarse por la Cámara en su Reglamento. También a las minorías se dirigían propuestas como las de conceder nuevos derechos a las minorías de oposición: creación de Comisiones de estudio, igualdad de tiempo de palabra entre mayoría y oposición en los debates, etc.

\section{A MODO DE CONCLUSIÓN}

En definitiva, de forma un tanto sorprendente y por la mínima mayoría, lo cierto es que la Constitución francesa de 1958 ha registrado un cambio cuyas consecuencias últimas todavía estamos lejos de prever. Y que, previsiblemente, deberá verse completado por medidas como las observadas en el apartado anterior, así como por otras propuestas por la Comisión Balladur y cuya puesta en práctica no depende de la Constitución, sino de simples reformas legales.

Ciertamente, la mayoría de un solo voto y la oposición frontal de casi toda la izquierda permitirían augurar un futuro polémico a las nuevas disposiciones. Sin embargo, y al margen de que la mayoría, aunque mínima, sea suficiente (como se ha recordado durante este debate, un solo voto permitió, en 1875, la aprobación de la "enmienda Wallon", que por primera vez proclamó la III República) previsiblemente muchas de ellas serán irreversibles. Algo que, en cualquier caso, podrá ser objeto de futuras crónicas.

ABSTRACT.- In 2008, two new constitutional laws amending the 1958 French Constitution have been passed by the French Parliament. The first is aimed at adapting the Constitution to the new European framework, after the failure of the Constitutional Treaty and the adoption of the Lisbonne Treaty. But the second is far more relevant: in fact, it implies a modernization of the French institutional framework, by reinforcing the position of Parliament, setting up new limits and constraints to Presidential powers, and creating new guarantees of fundamental rights, among other changes. 